\title{
Aged Wine and Symphonic Perfection
}

\author{
Ahmed Chaudhry $\cdot$ Malek G. Massad
}

Published online: 30 November 2009

(C) Société Internationale de Chirurgie 2009

In this issue, Lin and Pai evaluate the impact of increasing surgeon age on in-hospital mortality associated with coronary artery bypass grafting (CABG) surgery. They conclude that age and experience afforded to a surgeon by seniority is directly linked to improved outcomes [1].

The concept of experience and improved outcomes is not new, nor is it novel to the operative environment. In many industries, experience has been shown to have an impact on outcomes of performance. Before we analyze the surgical model with age-related outcome bias, let us consider an example in recent aviation history where experience was deemed to lead to a better outcome. The landing of US Air flight 1549 in New York's Hudson River earlier this year by Captain Sullenberger, who had 19,000 hours of flight experience in the cockpit, was directly related to the fact that he was able to perform an exacting landing in the face of engine failure, thereby saving countless lives.

It is no mystery that wine aged to perfection indeed tastes better. It is also amply evident that more experienced surgeons perform complex operations with better outcomes, whereas younger surgeons may have less than desirable outcomes even after routine procedures owing to their lack of experience. One of the underlying reasons is perhaps the quality of training a surgeon receives during residency or fellowship. It has been noted that although trainees may have been exposed to a lot of procedures their skill is commensurate with that of procedures they have actually performed. It is also a duly noted fact that senior surgical faculty are better at training residents because they

A. Chaudhry · M. G. Massad ( $\square)$

Division of Cardiothoracic Surgery, The University of Illinois

at Chicago, 840 S. Wood Street, CSB 417 (MC 958), Chicago,

IL 60612, USA

e-mail: mmassad@uic.edu know that a technical error made by a resident can be rectified by the senior surgeon's experience. Many skilled professors of surgery with thousands of operations behind them allow the resident to do more in the operating room because they know that they can fix any mistakes made by the resident and still have a good outcome. The junior surgeons are more resistant to such liberality in the teaching setting because they may not be skilled enough to rectify technical mistakes made by the residents.

Yet, as mentioned before, most clinical setups have both senior and junior surgeons performing the operations. When a difficult operation is scheduled by a junior surgeon, she or he is afforded the courtesy of being assisted by a "senior partner," thus ensuring a favorable outcome. This is not to ignore an important argument that must be taken into consideration while analyzing surgeons' age-related outcomes - the fact that senior surgeons usually have the liberty to be selective about whom they operate on and may have the clout to choose the lowest-risk patients, thus allowing them access to a patient pool that is likely to have better outcomes. Consequently, the "junior surgeon" gets the less favorable cases; and between his or her lack of experience and the high-risk cases, it is a setup staged to result in less than desirable outcomes.

It is further noted that senior surgeons, based on their experience, have developed operative logistical maneuvers over the years that allow them greater reproducibility of the technical skills that are required when performing simple or even complex operations. Their movements and operative steps are more consistent and more fluid than those of the junior surgeon, whose technique is still evolving. When considering a CABG procedure, one could view this complex operation as an array of serial successive steps that are added together systematically and that would include opening the chest, harvesting the bypass conduits, 
establishing cannulation, performing the distal and proximal anastomoses, going on and coming off cardiopulmonary bypass, and affording adequate myocardial preservation. This symphonic performance has been mastered by the senior surgeons through their years of experience; it was learned through consistency and avoidance of near-miss events, thereby bringing the operative mortality in low-risk cases to just over $1 \%$. Whereas a senior surgeon can perform each part of the operation flawlessly over and over again, the junior surgeon may not have perfected every step of the operation; and small mistakes or errors during any step of the operation may translate into higher overall complication and death rates.

That said, it is not necessarily true that younger surgeons are a detriment to the surgical patient pool. If anything, they usually bring to the operating room newer techniques that may not be in the armamentarium of the senior surgeon, who has been performing the exact same operation for years; and because the senior surgeon has perfected that technique to the level of symphonic perfection, she or he may not be receptive to the new ideas, techniques, and zeal exhibited by the junior surgeon. It is well known that freshly trained surgeons are better skilled at performing the minimally invasive procedures to which they were exposed during their training. Just as the senior surgeon may show a junior counterpart how better to perform a complex operation such as an Ivor-Lewis or a transhiatal esophagectomy, an aortic root replacement, a thoracoabdominal aneurysm repair, or a redo open cardiac procedure, the junior surgeon may have better skill and understanding of a minimally invasive procedure with which he or she is comfortable performing.

There will always be variability in the age, experience, annual case volume, training and background, and patient selection and stratification per surgeon in every established practice at any given time. No surgeon is born de novo with thousands of operative procedures in their log. Although some surgeons indeed have gifted hands, as we have all seen, most surgeons perform their thousands of operations one case at a time and learn from any possible mistakes; yet there is never a guarantee that a surgeon with tremendous skill and experience performing his or her ten-thousandth simple operation can always produce a good outcome.

There is much left to chance in surgery every single day and in every operating room, irrespective of the experience, staffing, equipment, and consistency of the operating environment, as much can go wrong regardless of the extent of the surgeon's experience. Furthermore, although experience does matter in all disciplines, be it surgery or flying commercial planes, sometimes it is better to be lucky than good in the surgical world. One is always striving to become a better surgeon; and analyzing and ratiocinating over each step of each procedure performed affords the surgeon constant quality improvement with a resolute commitment to practice-based learning. This process affords our patients the best chance at a favorable outcome each and every day.

\section{Reference}

1. Lin HC, Pai HT (2009, in press) Increasing surgeon age is associated with decreasing in-hospital mortality in coronary artery bypass surgery. World J Surg. doi:10.1007/s00268-009-0291-0 